\title{
Linear temperature dependence of resistivity and change in the Fermi surface at the pseudogap critical point of a high- $T_{c}$ superconductor
}

\author{
R. Daou ${ }^{1 \star}$, Nicolas Doiron-Leyraud ${ }^{1 \star}$, David LeBoeuf ${ }^{1}$, S. Y. Li ${ }^{1}$, Francis Laliberté ${ }^{1}$, \\ Olivier Cyr-Choinière' ${ }^{1}$, Y. J. Jo², L. Balicas ${ }^{2}$, J.-Q. Yan ${ }^{3}$, J.-S. Zhou ${ }^{3}$, J. B. Goodenough ${ }^{3}$ and \\ Louis Taillefer ${ }^{1,4 \dagger}$
}

\begin{abstract}
A fundamental question for high-temperature superconductors is the nature of the pseudogap phase, which lies between the Mott insulator at zero doping and the Fermi liquid at high doping $p$ (refs 1,2). Here we report on the behaviour of charge carriers near the zero-temperature onset of this phase, namely at the critical doping $p^{*}$, where the pseudogap temperature $\boldsymbol{T}^{*}$ goes to zero, accessed by investigating a material in which superconductivity can be fully suppressed by a steady magnetic field. Just below $p^{*}$, the normal-state resistivity and Hall coefficient of $\mathrm{La}_{1.6-x} \mathrm{Nd}_{0.4} \mathrm{Sr}_{x} \mathrm{CuO}_{4}$ are found to rise simultaneously as the temperature drops below $\mathrm{T}^{*}$, suggesting a change in the Fermi surface with a large associated drop in conductivity. At $\boldsymbol{p}^{*}$, the resistivity shows a linear temperature dependence as the temperature approaches zero, a typical signature of a quantum critical point ${ }^{3}$. These findings impose new constraints on the mechanisms responsible for inelastic scattering and Fermi-surface transformation in theories of the pseudogap phase ${ }^{1,4-8}$.
\end{abstract}

At low hole doping $p$, high-transition-temperature (high- $T_{\mathrm{c}}$ ) superconductors are doped Mott insulators, strongly correlated metals characterized by a low carrier density $n$ equal to the concentration of doped holes. Indeed, Hall-effect measurements on $\mathrm{La}_{2-x} \mathrm{Sr}_{x} \mathrm{CuO}_{4}$ (LSCO) at $x=p<0.05$ yield a Hall number $n_{\mathrm{H}} \equiv V / e R_{\mathrm{H}}$ equal to $p$ at low temperature ${ }^{9}$, where $R_{\mathrm{H}}$ is the Hall coefficient, $e$ is the electron charge and $V$ is the volume per $\mathrm{Cu}$ atom. At high doping, however, these materials are Fermi liquids, metals characterized by a well-defined coherent three-dimensional Fermi surface ${ }^{10}$ and a resistivity $\rho$ that grows quadratically with temperature ${ }^{11}: \rho \sim T^{2}$. In this regime, the Fermi surface is a large cylinder containing $1+p$ holes ${ }^{10}$, so the carrier density is high, given by $n=1+p$. At $p \approx 0.25$, low-temperature measurements on $\mathrm{Tl}_{2} \mathrm{Ba}_{2} \mathrm{CuO}_{6+y}$ yield $n_{\mathrm{H}}=1+p$ (ref. 12 ). These findings naturally beg the following question: How do the electrons in copper oxide superconductors go from one state to the other?

This is intimately tied to the question of the nature of the 'pseudogap phase', this enigmatic region of the doping phase diagram present in all high- $T_{\mathrm{c}}$ superconductors below a crossover temperature $T^{*}$ (ref. 2). Here we investigate the $T=0$ onset of this pseudogap phase by measuring the transport properties of $\mathrm{La}_{1.6-x} \mathrm{Nd}_{0.4} \mathrm{Sr}_{x} \mathrm{CuO}_{4}$ (Nd-LSCO), a material whose relatively low

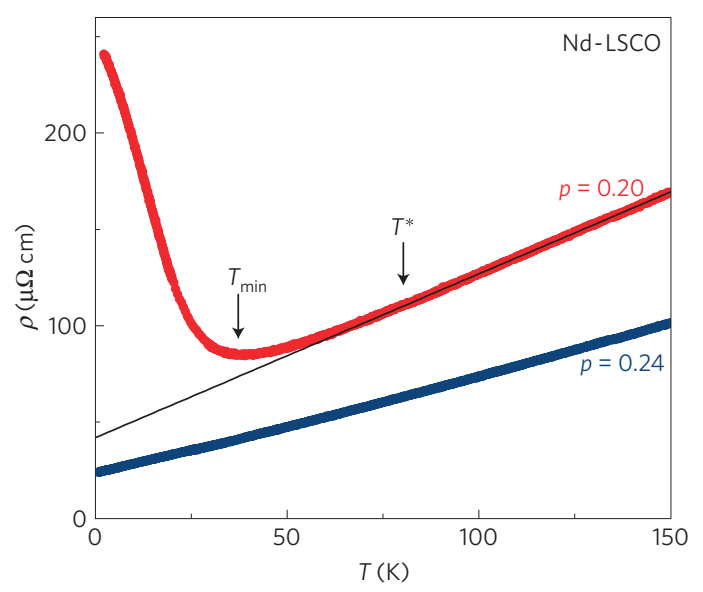

Figure 1 | Normal-state resistivity. In-plane electrical resistivity $\rho(T)$ of $\mathrm{Nd}$-LSCO as a function of temperature, at $p=0.20$ and 0.24 , measured in a magnetic field strong enough to fully suppress superconductivity (see Supplementary Information). The black line is a linear fit to the $p=0.20$ data between 80 and $300 \mathrm{~K}$. Below a temperature $T^{*}=80 \mathrm{~K}, \rho(T)$ deviates from its linear- $T$ behaviour at high temperature and develops a pronounced upturn at low temperature, with a minimum at $T_{\min }=37 \mathrm{~K}$. By contrast, $\rho(T)$ at $p=0.24$ shows no upturn down to the lowest temperature.

maximal $T_{\mathrm{c}}$ makes it possible to suppress superconductivity entirely with a steady magnetic field.

In Fig. 1, we show the normal-state resistivity $\rho(T)$ of Nd-LSCO at a doping $p=0.20$. Above a temperature $T^{*}=80 \mathrm{~K}, \rho(T)$ shows the linear temperature dependence characteristic of all hole-doped copper oxides. Below this temperature, it deviates upwards and develops an upturn visible even in zero field (see Supplementary Information, Fig. S1), with a minimum at $T_{\min }=37 \mathrm{~K}>T_{\mathrm{c}}=20 \mathrm{~K}$, in excellent agreement with early data in zero field ${ }^{13}$. By applying a magnetic field of $35 \mathrm{~T}$, we were able to track the upturn in $\rho(T)$ down to $1 \mathrm{~K}$, thus revealing a pronounced rise at low temperature (Fig. 1).

\footnotetext{
1Département de physique and RQMP, Université de Sherbrooke, Sherbrooke, Québec J1K 2R1, Canada, ${ }^{2}$ National High Magnetic Field Laboratory, Florida State University, Tallahassee, Florida 32310-3706, USA, ${ }^{3}$ Texas Materials Institute, University of Texas at Austin, Austin, Texas 78712, USA, ${ }^{4}$ Canadian Institute for Advanced Research, Toronto, Ontario M5G 1Z8, Canada. ${ }^{\star}$ These authors contributed equally to this work.

†e-mail: louis.taillefer@physique.usherbrooke.ca.
} 
a

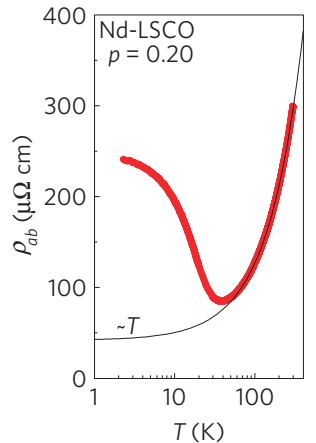

b

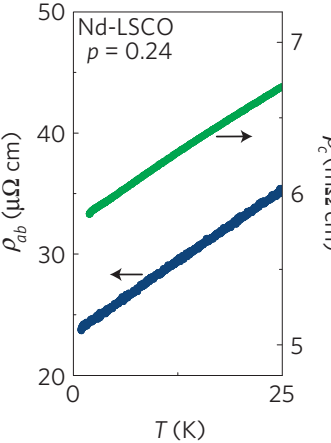

Figure $\mathbf{2}$ | In-plane and out-of-plane resistivities at low temperature. a, Semilog plot of the in-plane resistivity $\rho_{a b}(T)$ of Nd-LSCO at $p=0.20$. The black line is a linear fit above $80 \mathrm{~K}$. This shows that, after a rapid rise, the in-plane resistivity saturates at low temperature, in contrast to the weak logarithmic divergence observed in LSCO at $p<0.16$ (ref. 29).

b. Temperature dependence of the normal-state electrical resistivity of Nd-LSCO at $p=0.24 \approx p^{*}$, in the low-temperature regime. Both $\rho_{a b}$ and the out-of-plane resistivity $\rho_{c}$ show a linear temperature dependence down to the lowest measured temperature.

The absence of magneto-resistance (see Supplementary Information, Fig. S1) implies that the magnetic field simply serves to remove superconductivity and reveal the unaltered behaviour of the underlying normal state down to $T \approx 0$. The evolution with temperature is perfectly smooth, indicating a crossover as opposed to a transition. Most significantly, $\rho(T)$ saturates at low temperature (see Fig. 2a). This shows that the ground state is a metal and not an insulator, and that $T^{*}$ therefore marks the onset of a crossover from one metallic state to another. Note that the loss of conductivity is substantial, by a factor of approximately $\rho_{0} / \rho(T \rightarrow 0)=5.8$, where $\rho_{0}=245 \mu \Omega \mathrm{cm}$ is the resistivity measured at $T=1 \mathrm{~K}$ and $\rho(T \rightarrow 0)=42 \mu \Omega \mathrm{cm}$ is the value extrapolated linearly to $T=0$ from above $T^{*}$.

We identify $T^{*}$ as the onset of the pseudogap phase, following the standard definition: the temperature below which the in-plane resistivity $\rho_{a b}(T)$ starts to deviate from its linear- $T$ behaviour at high temperature ${ }^{2,14}$. (Note that the deviation can be either upwards, as in LSCO, or downwards, as in $\mathrm{YBa}_{2} \mathrm{Cu}_{3} \mathrm{O}_{y}$ (ref. 14), depending on the relative magnitude of inelastic and elastic (disorder) scattering at $T^{*}$; in $\mathrm{YBa}_{2} \mathrm{Cu}_{3} \mathrm{O}_{y}$, the copper oxide material with the lowest disorder scattering, the loss of inelastic scattering below $T^{*}$ is a much larger relative effect than in LSCO, hence the drop in $\rho_{a b}(T)$.) In Fig. 3, we plot $T^{*}$ as a function of doping in a $p-T$ phase diagram. Note that the magnitude of $T^{*}$ in Nd-LSCO is comparable to that found in other hole-doped copper oxides, pointing to a common origin (see Supplementary Information, Fig. S2 for a comparison with LSCO).

In Fig. 4, we present the Hall coefficient $R_{\mathrm{H}}(T)$ measured on the same crystal (with $p=0.20$ ), and compare it directly with $\rho(T)$. Both coefficients are seen to rise simultaneously, with $T_{\min }$ the coincident onset of their respective upturns. This is strong evidence that the cause of both upturns is a modification of the Fermi surface.

Let us now look at a slightly higher doping. Figures 1 and 4 respectively show $\rho(T)$ and $R_{\mathrm{H}}(T)$ measured on a second crystal, with $p=0.24$. The low-temperature behaviour has changed: $\rho(T)$ shows no sign of an upturn and $R_{\mathrm{H}}(T)$ is now constant below $25 \mathrm{~K}$, extrapolating to $R_{\mathrm{H}}=+0.45 \pm 0.05 \mathrm{~mm}^{3} \mathrm{C}^{-1}$ as $T \rightarrow 0$. The corresponding Hall number is $n_{\mathrm{H}}=1.3 \pm 0.15$, in good agreement with the carrier density $n=1+p=1.24$ expected for a large Fermi cylinder, and quantitatively consistent with measurements on $\mathrm{Tl}_{2} \mathrm{Ba}_{2} \mathrm{CuO}_{6+y}$ at $p=0.26$ and $T \rightarrow 0$, where $n_{\mathrm{H}}=1.3$ (ref. 12).

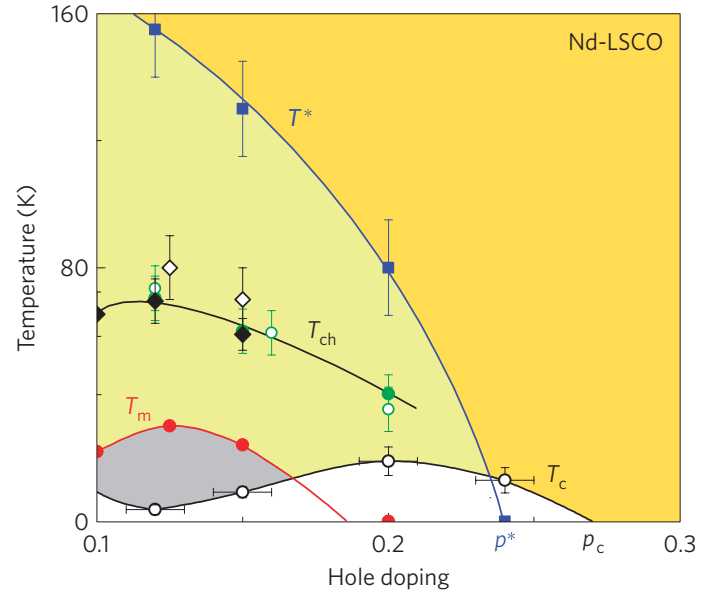

Figure 3 | Phase diagram. Temperature-doping phase diagram of Nd-LSCO showing the superconducting phase below $T_{\mathrm{c}}$ (open black circles) and the pseudogap region delineated by the crossover temperature $T^{*}$ (blue squares). Also shown is the region where static magnetism is observed below $T_{\mathrm{m}}$ (red circles) and charge order is detected below $T_{\mathrm{ch}}$ (black diamonds and green circles). These onset temperatures are respectively defined as the temperature below which (1) the resistance is zero, (2) the in-plane resistivity $\rho_{a b}(T)$ deviates from its linear dependence at high temperature, (3) an internal magnetic field is detected by zero-field muon spin relaxation and (4) charge order is detected by either $X$-ray diffraction or nuclear quadrupole resonance (NQR). All lines are guides to the eye. Values of $T_{\mathrm{C}}$ and error bars are given in Supplementary Information. $T^{*}$ is obtained from a fit to the $\rho_{a b}(T)$ data of ref. 20 for $p=0.12$ and 0.15 , and those reported here for $p=0.20$ and 0.24 (see Fig. 1 and Supplementary Information, Fig. S2, and Supplementary Information for fits and error bars). The blue line $p=0.20$ is made to end at $p=0.24$, thereby defining the critical doping where $T^{*}$ goes to zero as $p^{*}=0.24$. Experimentally, this point must lie in the range $0.20<p^{*} \leqslant 0.24$, because $\rho(T)$ remains linear down to the lowest temperature at $p=0.24$ (see Fig. $2 \mathrm{~b}$ ). $T_{\mathrm{m}}$ is obtained from the muon spin relaxation measurements of ref. 31 . The red line is made to end below $p=0.20$, as no static magnetism was detected at $p=0.20$ down to $T=2 \mathrm{~K} . T_{\text {ch }}$ is obtained from hard-X-ray diffraction on Nd-LSCO (filled black diamonds and error bars; ref. 22) and from resonant soft-X-ray diffraction on Eu-LSCO (open black diamonds and error bars; ref. 23). The onset of charge order has been found to coincide with the wipe-out anomaly in NQR at $T_{\mathrm{NQR}}$, reproduced here from ref. 21 (error bars quoted therein) for Nd-LSCO (filled green circles) and Eu-LSCO (open green circles).

By comparison, at $p=0.20$, the magnitude of $R_{\mathrm{H}}$ at $T \rightarrow 0$ yields $n_{\mathrm{H}}=0.3 \pm 0.05$. The change in the Hall number at $T \rightarrow 0$ between $p=0.24$ and $p=0.20$ is therefore $\Delta n_{\mathrm{H}}=1.0 \pm 0.2$ hole per $\mathrm{Cu}$ atom. If the Hall number is interpreted as a carrier density, these values are consistent with a crossover from a metal with a large hole-like Fermi surface at $p^{*}$ (where $n=1+p$ ) to a metal with a low density of holes below $p^{*}$ (where $n \approx p$ ).

In contrast to $p=0.20$, the electrical resistivity at $p=0.24$ shown in Fig. $2 \mathrm{~b}$ shows a monotonic temperature dependence down to $1 \mathrm{~K}$, linear as $T \rightarrow 0$. The absence of any anomaly demonstrates that $T^{*}=0$ at that doping. Therefore, the critical doping $p^{*}$ where the pseudogap line ends is located between $p=0.20$ and 0.24 , inside the region where superconductivity exists in zero field. For definiteness, in Fig. 3 we set it at $p^{*}=0.24$, although it could be slightly lower.

As shown in Fig. 2b, not only is the in-plane resistivity $\rho_{a b}(T)$ linear as $T \rightarrow 0$ at $p=0.24$, but so is the out-ofplane resistivity $\rho_{c}(T)$. Moreover, the fact that $R_{\mathrm{H}}(T)$ is flat at low temperature implies that the cotangent of the Hall angle, 


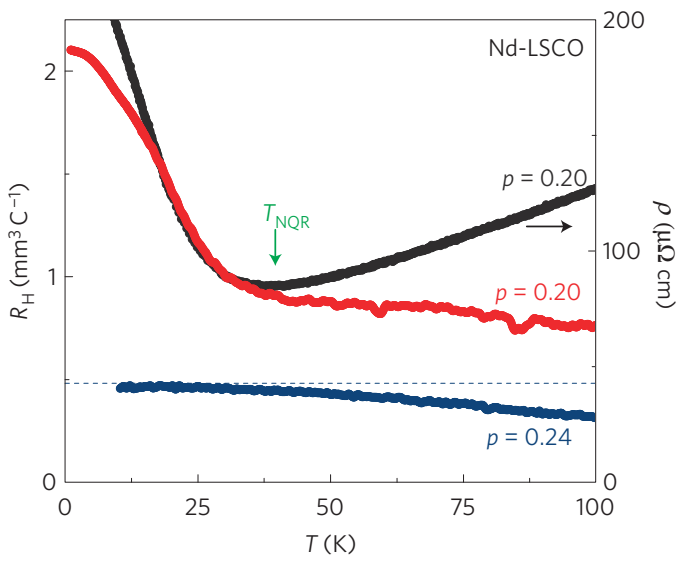

Figure 4 | Normal-state Hall coefficient. Hall coefficient $R_{H}(T)$ of $\mathrm{Nd}$-LSCO as a function of temperature for $p=0.20$ and 0.24 , measured in a magnetic field of $15 \mathrm{~T}$. Below $12 \mathrm{~K}$, the 0.20 data are in $33 \mathrm{~T}$, a magnetic field strong enough to fully suppress superconductivity (see Supplementary Information). The dashed blue horizontal line is the value of $R_{H}$ calculated for a large cylindrical Fermi surface enclosing $1+p$ holes, namely $R_{H}=V / e$ $(1+p)$, at $p=0.24$. At $p=0.20$, the rise in $R_{H}(T)$ at low temperature signals a modification of this large Fermi surface. The upturn is seen to coincide with a simultaneous upturn in $\rho(T)$ (reproduced in black from Fig. 1) and with the onset of charge order at $T_{N Q R}$ as detected by NQR (see the text and ref. 21).

$\cot \theta_{\mathrm{H}}(T) \sim \rho_{a b}(T) / R_{\mathrm{H}}(T)$, is also linear at low temperature. We infer that a single anomalous scattering process dominates the electron-electron correlations at low temperature at $p^{*}$ (or just above). This shows that the Fermi-liquid behaviour observed at $p=0.3$ (in LSCO), where $\rho_{a b}(T) \sim T^{2}$ below $T \approx 50 \mathrm{~K}$ (ref. 11 ), breaks down just before the onset of the pseudogap phase at $p^{*}$. This kind of 'non-Fermi-liquid' behaviour, whereby $\rho(T) \sim T$ as $T \rightarrow 0$, has typically been observed in heavy-fermion metals at the quantum critical point where the onset temperature for antiferromagnetic order goes to zero ${ }^{3}$. It is also consistent with the marginal-Fermi-liquid description of cuprates ${ }^{15}$.

In summary, our experimental findings offer compelling evidence that the pseudogap phase ends at a $T=0$ critical point $p^{*}$ located below the onset of superconductivity (at $p_{\mathrm{c}} \approx 0.27$ ), in agreement with previous but more indirect evidence from other hole-doped copper oxides ${ }^{16}$. Moreover, they impose two strong new constraints on theories of the pseudogap phase: (1) its onset below $p^{*}$ modifies the large Fermi surface characteristic of the overdoped metallic state; (2) quasiparticle scattering at $p^{*}$ is linear in temperature as $T \rightarrow 0$.

The existence of a quantum critical point is consistent with two kinds of theory of the pseudogap phase. The first kind invokes the onset of an order, with some associated broken symmetry ${ }^{6-8}$. Because $T^{*}$ marks a crossover and not a sharp transition, this order is presumably short range or fluctuating. In the electrondoped copper oxides, for example, the pseudogap phase has been interpreted as a fluctuating precursor of the long-range antiferromagnetic order that sets in at lower temperature ${ }^{17}$, and the signatures of the pseudogap critical point in transport are similar to those found here: a linear- $T$ resistivity as $T \rightarrow 0$ (ref. 18) and a sharp change in $R_{\mathrm{H}}(T=0)$ (ref. 19). For Nd-LSCO and LSCO, an analogous scenario would be 'stripe' fluctuations, as a precursor to the static spin and charge modulations observed at lower temperature $\mathrm{e}^{20}$. Note that in Nd-LSCO at $p=0.20$ the onset of the upturn in $\rho(T)$ and $R_{\mathrm{H}}(T)$ at $T_{\min }=37 \mathrm{~K}$ coincides with the loss of NQR intensity at $T_{\mathrm{NQR}}=40 \pm 6 \mathrm{~K}$ (ref. 21) (see Fig. 4). In Nd-LSCO at $p=0.15$, this so-called 'wipe-out' anomaly in NQR at
$T_{\mathrm{NQR}}=60 \pm 6 \mathrm{~K}$ (ref. 21) was shown to coincide with the onset of charge order measured via hard-X-ray diffraction, at $T_{\mathrm{ch}}=62 \pm 5 \mathrm{~K}$ (ref. 22) (see Fig. 3). Direct evidence of a charge modulation via resonant soft-X-ray diffraction was reported recently for the closely related material Eu-LSCO, with $T_{\mathrm{ch}}=70 \pm 10 \mathrm{~K}$ at $p=0.15$ (ref. 23), whereas $T_{\mathrm{NQR}}=60 \pm 6 \mathrm{~K}$ (ref. 21) in Eu-LSCO at $p=0.16$ (see Fig. 3). Clearly, the upturn in $\rho(T)$ is correlated with the onset of charge order in these two materials. While the correlation between $T_{\mathrm{NQR}}$ and $T_{\text {min }}$ has been noted previously ${ }^{20}$, the mechanism causing the upturn in $\rho(T)$ remained unclear. Our data show that the mechanism is a change in Fermi surface, and the positive rise in $R_{\mathrm{H}}(T)$ imposes a strong constraint on the topology of the resulting Fermi surface. An additional constraint comes from the fact that $R_{\mathrm{H}}(T)$ drops to negative values near $p=1 / 8$, not only in Nd-LSCO (ref. 13) and other materials with 'stripe' order ${ }^{24,25}$, but also in $\mathrm{YBa}_{2} \mathrm{Cu}_{3} \mathrm{O}_{y}$ (ref. 26).

Recent calculations of the Fermi-surface reconstruction caused by stripe order are consistent with a negative $R_{\mathrm{H}}$ near $p=1 / 8$ in that spin stripes tend to generate an electron pocket in the Fermi surface $^{27}$. Interestingly, charge stripes do not ${ }^{27}$, and this might explain the positive rise in $R_{\mathrm{H}}$ seen at higher doping, provided that stripe order involves predominantly charge order at high doping (in line with the fact that charge order sets in at a higher temperature than spin order ${ }^{20,21}$ ).

In the other kind of theory of the pseudogap phase, the critical point reflects a $T=0$ transition from small hole pockets, characteristic of a doped Mott insulator, to a large hole pocket, without symmetry breaking ${ }^{4,5}$. Recent work suggests that the quasiparticle scattering rate above such a critical point may indeed grow linearly with temperature ${ }^{28}$. Although calculations are needed to confirm this, a change in carrier density from $n \approx p$ to $n=1+p$ would seem natural in this kind of scenario. However, it is more difficult to see what could cause the negative values of $R_{\mathrm{H}}(T \rightarrow 0)$ near $p=1 / 8$. It seems that stripe order or fluctuations would have to be invoked as a secondary instability inside the pseudogap phase, with an onset in doping that would be essentially simultaneous with $p^{*}$ in the case of Nd-LSCO.

We end by comparing our results qualitatively with those of previous high-field studies on LSCO. The resistivity shows very similar features at high temperature: linear $T$ above $T^{*}$ (ref. 14) and an upturn below $T^{*}$ (ref. 29). The Hall coefficient of LSCO (ref. 30), on the other hand, has a more subtle and complex evolution with doping than that presented here for Nd-LSCO, which makes it harder to pinpoint $p^{*}$ using the same criteria as we have used above. Nonetheless, it seems likely that the same fundamental mechanisms are responsible for both the linear- $T$ resistivity and the resistivity upturns, and for the onset of the pseudogap at $T^{*}$, in both LSCO and Nd-LSCO.

\section{Methods}

Single crystals of $\mathrm{La}_{2-y-x} \mathrm{Nd}_{y} \mathrm{Sr}_{x} \mathrm{CuO}_{4}$ (Nd-LSCO) were grown with a $\mathrm{Nd}$ content $y=0.4$ using a travelling-float-zone technique and cut from boules with nominal Sr concentrations $x=0.20$ and 0.25 . The actual doping $p$ of each crystal was estimated from its $T_{\mathrm{c}}$ and $\rho(250 \mathrm{~K})$ values compared with published data, giving $p=0.20 \pm 0.005$ and $0.24 \pm 0.005$, respectively. The resistivity $\rho$ and Hall coefficient $R_{\mathrm{H}}$ were measured at the National High Magnetic Field Laboratory (NHMFL) in Tallahassee in steady magnetic fields up to $35 \mathrm{~T}$ and in Sherbrooke in steady fields up to $15 \mathrm{~T}$. The field was always applied along the $c$ axis. Neither $\rho$ nor $R_{\mathrm{H}}$ showed any field dependence up to the highest fields. More details are available in Supplementary Information.

\section{Received 5 June 2008; accepted 24 September 2008; published online 2 November 2008}

\section{References}

1. Lee, P. A., Nagaosa, N. \& Wen, X.-G. Doping a Mott insulator: Physics of high-temperature superconductivity. Rev. Mod. Phys. 78, 17-85 (2006).

2. Timusk, T. \& Statt, B. The pseudogap in high-temperature superconductors: An experimental survey. Rep. Prog. Phys. 62, 61-122 (1999). 
3. v Löhneysen, H., Rosch, A., Vojta, M. \& Wölfle, P. Fermi-liquid instabilities at magnetic quantum phase transitions. Rev. Mod. Phys. 79, 1015-1075 (2007).

4. Yang, K.-Y., Rice, T. M. \& Zhang, F.-C. Phenomenological theory of the pseudogap state. Phys. Rev. B 73, 174501 (2006).

5. Haule, K. \& Kotliar, G. Avoided criticality in near-optimally doped high-temperature superconductors. Phys. Rev. B 76, 192503 (2007).

6. Kivelson, S. A. et al. How to detect fluctuating stripes in the high-temperature superconductors. Rev. Mod. Phys. 75, 1201-1241 (2003).

7. Chakravarty, S., Laughlin, R. B., Morr, D. K. \& Nayak, C. Hidden order in the cuprates. Phys. Rev. B 63, 094503 (2001).

8. Varma, C. Non-Fermi-liquid states and pairing instability of a general model of copper oxide metals. Phys. Rev. B 55, 14554-14580 (1997).

9. Ando, Y. et al. Evolution of the Hall coefficient and the peculiar electronic structure of the cuprate superconductors. Phys. Rev. Lett. 92, 197001 (2004).

10. Hussey, N. E. et al. Observation of a coherent three-dimensional Fermi surface in a high-transition temperature superconductor. Nature 425, 814-817 (2003).

11. Nakamae, S. et al. Electronic ground state of heavily-overdoped non-superconducting $\mathrm{La}_{2-x} \mathrm{Sr}_{x} \mathrm{CuO}_{4}$. Phys. Rev. B 68, 100502 (2003).

12. Mackenzie, A. P. et al. Normal-state magnetotransport in superconducting $\mathrm{Tl}_{2} \mathrm{Ba}_{2} \mathrm{CuO}_{6+y}$ to millikelvin temperatures. Phys. Rev. B 53, 5848-5855 (1996).

13. Nakamura, Y. \& Uchida, S. Anisotropic transport properties of single-crystal $\mathrm{La}_{2-y-x} \mathrm{Nd}_{y} \mathrm{Sr}_{x} \mathrm{CuO}_{4}$ : Effect of the structural phase transition. Phys. Rev. B 46, 5841-5844 (1992).

14. Ando, Y. et al. Electronic phase diagram of high- $T_{\mathrm{c}}$ cuprate superconductors from a mapping of the in-plane resistivity curvature. Phys. Rev. Lett. 93, 267001 (2004).

15. Varma, C. M. et al. Phenomenology of the normal state of $\mathrm{Cu}-\mathrm{O}$ high-temperature superconductors. Phys. Rev. Lett. 63, 1996-1999 (1989).

16. Tallon, J. L. \& Loram, J. W. The doping dependence of $T^{*}-$ what is the real high- $T_{\mathrm{c}}$ phase diagram? Physica C 349, 53-68 (2001).

17. Motoyama, E. M. et al. Spin correlations in the electron-doped high-transition-temperature superconductor $\mathrm{Nd}_{2-x} \mathrm{Ce}_{x} \mathrm{CuO}_{4 \pm \delta}$. Nature 445, 186-189 (2007).

18. Fournier, P. et al. Insulator-metal crossover near optimal doping in $\mathrm{Pr}_{2-x} \mathrm{Ce}_{x} \mathrm{CuO}_{4}$ : anomalous normal-state low-temperature resistivity. Phys. Rev. Lett. 81, 4720-4723 (1998).

19. Dagan, Y. et al. Evidence for a quantum phase transition in $\operatorname{Pr}_{2-x} \mathrm{Ce}_{x} \mathrm{CuO}_{4-\delta}$ from transport measurements. Phys. Rev. Lett. 92, 167001 (2004).

20. Ichikawa, N. et al. Local magnetic order vs superconductivity in a layered cuprate. Phys. Rev. Lett. 85, 1738-1741 (2000).

21. Hunt, A. W. et al. Glassy slowing of stripe modulation in $(\mathrm{La}, \mathrm{Eu}, \mathrm{Nd})_{2-x}(\mathrm{Sr}, \mathrm{Ba})_{x} \mathrm{CuO}_{4}: \mathrm{A}^{63} \mathrm{Cu}$ and ${ }^{139} \mathrm{La} \mathrm{NQR}$ study down to $350 \mathrm{mK}$. Phys. Rev. B 64, 134525 (2001).
22. Niemöller, T. et al. Charge stripes seen with $\mathrm{X}$-rays in $\mathrm{La}_{1.45} \mathrm{Nd}_{0.4} \mathrm{Sr}_{0.15} \mathrm{CuO}_{4}$. Eur. Phys. J. B 12, 509-513 (1999).

23. Fink, J. et al. Charge order in $\mathrm{La}_{1.8-x} \mathrm{Eu}_{0.2} \mathrm{Sr}_{x} \mathrm{CuO}_{4}$ studied by resonant soft X-ray diffraction. Preprint at <http://arxiv.org/abs/0805.4352> (2008).

24. Adachi, T., Noji, T. \& Koike, Y. Crystal growth, transport properties, and crystal structure of the single-crystal $\mathrm{La}_{2-x} \mathrm{Ba}_{x} \mathrm{CuO}_{4}(x=0.11)$. Phys. Rev. B 64, 144524 (2001).

25. Takeshita, $\mathrm{N}$ et al. Giant anisotropic pressure effect on superconductivity within the $\mathrm{CuO}_{2}$ plane of $\mathrm{La}_{1.64} \mathrm{Eu}_{0.2} \mathrm{Sr}_{0.16} \mathrm{CuO}_{4}$ : Strain control of stripe criticality. J. Phys. Soc. Jpn. 73, 1123-1126 (2004).

26. LeBoeuf, D. et al. Electron pockets in the Fermi surface of hole-doped high- $T_{\mathrm{c}}$ superconductors. Nature 450, 533-536 (2007).

27. Millis, A. J. \& Norman, M. R. Antiphase stripe order as the origin of electron pockets observed in 1/8-hole-doped cuprates. Phys. Rev. B 76, 220503 (2007).

28. Ossadnik, M., Honerkamp, C., Rice, T. M. \& Sigrist, M. Breakdown of Landau theory in overdoped cuprates near the onset of superconductivity. Preprint at $<$ http://arxiv.org/abs/0805.3489> (2008)

29. Boebinger, G. S. et al. Insulator-to-metal crossover in the norma state of $\mathrm{La}_{2-x} \mathrm{Sr}_{x} \mathrm{CuO}_{4}$ near optimum doping. Phys. Rev. Lett. 77 , 5417-5420 (1996).

30. Balakirev, F. F. et al. Fermi surface reconstruction at optimum doping in high- $T_{\mathrm{c}}$ superconductors. Preprint at $<$ http://arxiv.org/abs/0710.4612> (2007).

31. Nachumi, B. et al. Muon spin relaxation study of the stripe phase order in $\mathrm{La}_{1.6-x} \mathrm{Nd}_{0.4} \mathrm{Sr}_{x} \mathrm{CuO}_{4}$ and related 214 cuprates. Phys. Rev. B 58, 8760-8772 (1998).

\section{Acknowledgements}

We thank K. Behnia, A. Chubukov, P. Coleman, Y.B. Kim, S.A. Kivelson, G. Kotliar, K. Haule, G.G. Lonzarich, A.J. Millis, M.R. Norman, C. Proust, T.M. Rice, S. Sachdev, T. Senthil, H. Takagi and A.-M.S. Tremblay for discussions, and J. Corbin for his assistance with the experiments. L.T. acknowledges support from the Canadian Institute for Advanced Research and funding from NSERC, FQRNT, and a Canada Research Chair. L.B. was supported by NHMFL-UCGP and Y.J.J. by the NHMFL-Schuller fellow program. J.S.Z. and J.B.G. were supported by an NSF grant. The NHMFL is supported by an NSF grant and the State of Florida.

\section{Additional information}

Supplementary Information accompanies this paper on www.nature.com/naturephysics. Reprints and permissions information is available online at http://npg.nature.com/ reprintsandpermissions. Correspondence and requests for materials should be addressed to L.T. 